\title{
Pas si simple! La place du PS dans l'interlangue d'apprenants anglophones avancés
}

\section{Il ne faut pas se fier à l'aspect}

Le développement du temps et de l'aspect en langue seconde retient l'attention des acquisitionnistes depuis une vingtaine d'années. Deux volumes récents (Bardovi-Harlig 2000, Salaberry \& Shirai 2002), tentant de faire le point sur la question, montrent combien le domaine est porteur. Un modèle, développé par Andersen à partir de 1986, s’est avéré des plus populaire ; il postule un développement aspectuo-temporel linéaire en huit étapes :

\begin{tabular}{l|l|l|l|l}
\hline & $\begin{array}{l}\text { États } \\
\text { 'avoir' }\end{array}$ & $\begin{array}{l}\text { Activités } \\
\text { jouer' }\end{array}$ & $\begin{array}{l}\text { Événements téliques } \\
\text { 'enseigner' }\end{array}$ & $\begin{array}{l}\text { Événements ponctuels } \\
\text { 'briser' }\end{array}$ \\
\hline 1 & tiene & juega & enseña & se parte \\
\hline 2 & tiene & juega & enseña & se partió \\
\hline 3 & tenía & juega & enseña & se partió \\
\hline 4 & tenía & jugaba & enseño & se partió \\
\hline 5 & tenía & jugaba & $\begin{array}{l}\text { enseño } \\
\text { enseñeba }\end{array}$ & se partió \\
\hline 6 & tenía & $\begin{array}{l}\text { jugaba } \\
\text { jugó }\end{array}$ & $\begin{array}{l}\text { enseño } \\
\text { enseñeba }\end{array}$ & se partió \\
\hline 7 & tenía & $\begin{array}{l}\text { jugaba } \\
\text { jugó }\end{array}$ & $\begin{array}{l}\text { enseño } \\
\text { enseñeba }\end{array}$ & $\begin{array}{l}\text { se partió } \\
\text { se partia }\end{array}$ \\
\hline 8 & $\begin{array}{l}\text { tenía } \\
\text { tuvo }\end{array}$ & $\begin{array}{l}\text { jugaba } \\
\text { jugó }\end{array}$ & $\begin{array}{l}\text { enseño } \\
\text { enseñeba }\end{array}$ & $\begin{array}{l}\text { se partió } \\
\text { se partia }\end{array}$ \\
\hline
\end{tabular}

Tableau 1: Séquence de développement des marqueurs de morphologie verbale (adaptation d'Andersen 1991, 314)

Au niveau 1, ni le temps passé ni l'aspect ne sont indiqués et une forme de base, le présent (PRES), est utilisée. Le niveau 2 voit l'apparition du prétérit (en italiques dans le tableau) uniquement avec des verbes ponctuels alors que les verbes statifs émergent à l'imparfait (IMP) au niveau 3 (en gras dans le tableau). Au niveau 4, un marquage du passé existe dans toutes les catégories, le prétérit s'étendant aux accomplissements et l'imparfait aux activités. A partir du niveau 5, la morphologie verbale commence progressivement à marquer toutes les catégories de verbes jusqu’à ce qu'au

1 La classification des syntagmes verbaux s’inspire des quatre catégories de Vendler (1967): states, activities, accomplishments et achievements. 
niveau 8, les verbes statifs apparaissent finalement au prétérit, ce qui devrait représenter le développement ultime du système aspectuel.

On a soulevé plus en détail ailleurs (Labeau, à paraître) les problèmes théoriques de cette 'hypothèse de l'aspect': la nature hypothétique du développement dont seulement 4 étapes sont attestées chez les deux sujets étudiés (Andersen 1991, 313-4), la validité des affinités une fois que tout marqueur temporel s'applique à tout type de verbe (Kihlstedt 1998, 43ss), la persistance de " petits pépins » (non native glitches) au niveau 8 (Andersen 1991, 316)... La recherche de terrain (Salaberry 2000 pour l'espagnol, Labeau 2002 pour le français) montre également que le développement aspectuo-temporel des apprenants d'instruction élevée (pour une définition de ce concept, voir Bartning 1997) ne correspond pas à ce modèle linéaire. On a suggéré que l'acquisition des temps du passé français suivît un mouvement de sous-application, surgénéralisation et régression (Salaberry 2000) avec stabilisation progressive ${ }^{2}$ autour des normes natives. Conscient des limites de son hypothèse, Andersen l'a d'ailleurs modifiée à plusieurs reprises et la dernière mouture de l'hypothèse (Andersen 2002) suggère 6 facteurs à étudier pour rendre compte du développement aspectuo-temporel. En plus de l'aspect lexical, il suggère d'y intégrer le type d'événement (les marqueurs du passé porteront d'abord sur des événements uniques, puis sur les événements habituels et répétitifs), la distinction entre réel et irréel (les emplois à valeurs d'irréel apparaîtront tardivement), la pragmatique (des fonctions comme l'IMP de politesse seront tardivement acquises), l'ancrage dans le premier plan ou l'arrière-plan (les marqueurs du passé porteront d'abord sur les verbes du premier plan) et la structure du discours. On ne peut qu'accueillir favorablement cet élargissement indispensable de l'hypothèse. Toutefois, il pourrait être nécessaire d'y ajouter - au moins en français - une dimension stylistique.

\section{Il était une fois... le passé simple}

Le français est riche en formes verbales susceptibles d'exprimer le passé. Leur fréquence et leurs utilisations ont cependant évolué au cours de l'histoire de la langue. Le passé simple (PS) est vu traditionnellement comme le tiroir de la narration par excellence (la "pierre d'angle du récit" de Barthes). Au cours du dernier demi-siècle, les linguistes ont néanmoins souvent débattu de la vitalité du PS. Selon certains, le PS est toujours très productif dans les champs limités de l'écrit et de l'oral préparé (Judge \& Healey 1983,108, Martin 1971,11, Pfister 1974, 401). D'autres (ex. Van Vliet 1983) prédisent sa disparition en invoquant l'argument que toutes les fonctions du PS peuvent être remplies par d'autres temps (Schogt 1964, 16).

2 On a évoqué le mouvement pendulaire (Labeau 2003 et 2004). 
Il serait par conséquent superflu d'enseigner le PS en français, langue étrangère (FLE) (Gougenheim et al. 1964)

La plupart des apprenants anglophones sont conscients de l'existence d'un " past historic » mais quelle est la place réelle de cette forme dans l'interlangue d'apprenants avancés au niveau licence? A part Labeau (2002, à paraître), aucune des études consacrées au développement du temps et de l'aspect en français langue seconde (Bergström 1995, Bardovi-Harlig \& Bergström 1996, Kihlstedt 1998, Salaberry 1998, Howard 2002) ne mentionne d'occurrences de PS. La présente étude sera donc de nature largement exploratoire: ce marqueur morphologique apparaîtra-t-il dans l'interlangue ${ }^{3}$ d'apprenants avancés ? Le cas échéant cette émergence exiget-elle un cotexte ou un contexte particulier?

\section{Un jour, mon PS viendra... ?}

Cette recherche est basée sur un corpus de narrations orales et écrites rassemblées auprès de francophones et d'apprenants anglophones qualifiés ${ }^{4}$ après la lecture du conte Cendrillon de Charles Perrault. Un conte de fées était jugé propice à l'emploi du PS : les productions littéraires de ce genre y ont recours ; les locuteurs natifs perçoivent le PS comme la marque du genre et utilisent le tiroir dans leurs propres narrations. ${ }^{5}$

En plus d'un groupe de contrôle de $13^{6}$ francophones ( $N$ pour 'natifs'), quatre groupes d'apprenants (désignés par A pour les 'apprenants, ${ }^{7}$ et B pour les 'bilingues') ont été pris en compte:

3 Suggérée par Selinker en 1972, la notion d'interlangue (interlanguage) peut être définie comme les grammaires intermédiaires que les apprenants construisent au cours de leur acquisition de la langue cible. La notion implique à la fois une dimension horizontale ou synchronique référant à l'interlangue que l'apprenant possède à un moment donné et une dimension verticale ou diachronique lié aux niveaux développementaux que l'apprenant traverse.

${ }^{4}$ Pour une définition du concept d’ « apprenant qualifié », voir Bartning 1997.

5 «Une histoire, ça doit être au passé historique. Ou bien personne ne sait que c’est une histoire. » (Robbe-Grillet Djinn 1981,51).

6 La disparité numérique entre les groupes s'explique ainsi : les productions des étudiants bilingues et de première année ont été rassemblées auprès de tous les étudiants entrants par l'auteur en septembre 2003 ; les autres données ont été recueillies auprès de volontaires en novembre 2003 par des étudiants de 2ème année dans le cadre du cours "Contemporary French » (LF2408) à Aston University. Le même protocole de recherche a été rigoureusement suivi. On a choisi de prendre en compte toutes les données disponibles car il aurait été arbitraire de supprimer certains éléments du corpus plutôt que d'autres.

7 Les apprenants sont identifiés par un premier numéro (1, 2 ou 4) indiquant leur niveau d'étude et de deux numéros d’identification personnelle. 
(1) 34 étudiants de première année pendant la semaine d’accueil à l'université;

(2) 13 étudiants de deuxième année qui avaient reçu un enseignement explicite de la forme dans leur cours de grammaire de première année;

(3) 16 étudiants de quatrième année susceptibles d'avoir acquis par ailleurs une certaine perception des conventions stylistiques adoptées par les francophones natifs au cours de leur année à l'étranger et

(4) 8 sujets bilingues, étudiants de première année, dont la situation personnelle (famille bilingue, séjour en pays francophone, école française) a permis une exposition au français en milieu naturel.

La moitié des sujets ont lu le texte en français (et donc au PS) et l'autre moitié l'a lu en anglais. ${ }^{8}$ Après 10 minutes de lecture, ils ont enregistré (10 min) en laboratoire de langue leur propre version de l'histoire, avant de rédiger une version écrite (20 $\mathrm{min})$. Les sujets ont finalement rempli un questionnaire portant sur leur expérience linguistique qui contenait en outre une liste de 10 verbes à mettre au PS.

Grâce à cette méthodologie, on espérait tester les questions de recherche suivantes:

(1) Quelle est la fréquence d'emploi du PS dans la production écrite, orale et métalinguistique d'apprenants qualifiés?

(2) La présence du PS dans l'input fourni entraîne-t-elle l'utilisation de la forme par les apprenants?

(3) Les impératifs du genre « conte de fées » ont-ils raison de la répugnance à utiliser le PS à l'oral?

(4) L’utilisation du PS est-elle productive ou formulaire ?

(5) Quel(s) facteur(s) amène(nt) à recourir au PS: des facteurs personnels (l'enseignement et / ou le séjour à l'étranger et /ou le contexte familial), des facteurs textuels ou encore stylistiques?

\section{Analyse des données}

\subsection{Fréquence d'emploi du PS}

On a dépouillé les narrations écrites et orales des quatre groupes d'apprenants et du groupe de contrôle et relevé les formes verbales conjuguées ou qui en tenaient lieu ${ }^{9}$. On n'a donc pris en compte les infinitifs et les participes ${ }^{10}$ qu'en emplois de forme de base :

8 La version originale de Charles Perrault ainsi que sa traduction anglaise ont été télédéchargées du site: http://www.chez.com/feeclochette/Perrault/cendrill.htm.

${ }^{9}$ Pour les narrations orales, on a adopté les conventions de transcription présentées dans Kihlstedt (1998). Dans le comptage, on n'a pas pris en compte les 
(1) Il faut que la fille faire le menage, la lessive, etc. (A109)

(2) Quand Cinderella arrivé le fils de roi decidé de danser avec Cinderella mais Cinderella doit partir de minuit. A la maison Cinderella était toujours parlé sur le nuit. Elle demandé aller encore ! Elle entendé très bien avec le fils de roi mais elle oubliér sur le minuit! Cinderella parti très vite est sa chaussere resté en bal! (A101)

On a également constitué une catégorie de formes indéterminées (répertoriées sous le signe '?') qui regroupe d'une part les formes créées et d'autre part les formes ambiguës, présentant des caractéristiques morphologiques de plusieurs tiroirs français et ne pouvant donc pas être classifiés de façon univoque:

(3) Cinderella dansaient avec le Prince et elle a rencontrait ses deux belles-soeurs. (A401)

(4) Puis et est habite au palace

Ce relevé a donné les proportions suivantes d'emploi du PS ; la première colonne indique les chiffres absolus, la seconde le pourcentage par rapport à la totalité des formes verbales prises en compte :

\begin{tabular}{|l|l|l|l|l|}
\hline & \multicolumn{2}{|l|}{ Narrations écrites } & \multicolumn{2}{l|}{ Narrations orales } \\
\hline $1^{\text {ère }}$ année & $23^{11}$ & 1.91 & 0 & 0 \\
\hline $2^{\text {ème }}$ année & 0 & 0 & $3^{12}$ & 0.53 \\
\hline $4^{\text {ème }}$ année & $7^{13}$ & 1.22 & $2^{14}$ & 0.23 \\
\hline Bilingues & $61^{15}$ & 19.55 & $68^{16}$ & 9.86 \\
\hline Locuteurs natifs & $329^{17}$ & 61.73 & $195^{18}$ & 25.26 \\
\hline
\end{tabular}

propositions avortées et en cas de répétition d'une forme due à des erreurs de performance, seule une occurrence a été retenue.

${ }^{10}$ Etant donné l'identité phonique des infinitifs (INF) et participes passés (PP) en -er qui constituent la majorité des verbes français, on a confondu ces deux catégories dans les comptages oraux.

${ }^{11}$ A101, A105, A107, A112, A116, A123, A125, A126, A127 en produisent chacun deux occurrences ; A106 et A132 une et A128, trois.

${ }^{12}$ L'enregistrement de A202 en offre 2 exemples et celui de A213 un seul.

${ }^{13}$ Ces occurrences apparaissent chez A406 (2), A407 (2), A410 (1) et A412.

${ }^{14}$ Une occurrence apparaît dans les enregistrements de A403 et A407.

${ }^{15}$ Tous les bilingues emploient au moins une fois (B01, B07) le PS et la proportion d'utilisation est la plus élevée (47,06\%) chez B02. Malgré cette aisance d'utilisation du PS, B02 semble manquer de vocabulaire métalinguistique et fournit des $\mathrm{PC}$ et des IMP pour les dix verbes à mettre au PS.

${ }^{16}$ Trois sujets ont recours au PS : B02, BO4 et B08, mais dans le cas de ce dernier, c'est une occurrence isolée. Le PS constitue 15,38\% des formes utilisées par B04 et $60,64 \%$ chez B02. 
Tableau 2 : Emploi du PS dans les corpus

Deux résultats semblent a priori étonnants: (1) le groupe de $1^{\text {ère }}$ année présente la fréquence d'emploi du PS la plus élevée des trois groupes d'apprenants alors qu'il est le moins avancé et (2) le PS apparaît à l'oral et pas à l'écrit, contexte pourtant jugé plus favorable, dans le corpus de $2^{\text {ème }}$. Une approche qualitative nous permettra de relativiser ces anomalies apparentes. Ainsi, dans les narrations écrites de $1^{\text {ère }}$ année, on trouve huit fois la formule « ils furent heureux et eurent beaucoup d'enfants » qui concluait la liste d'illustrations distribuées aux sujets pour les aider à se rappeler les péripéties principales de l'histoire. 16 des 23 occurrences de PS ne sont donc pas des emplois productifs mais du simple recopiage. Il reste donc les sept formes suivantes : fut (A106) [2], fit (A127), se mit (A128), donna (A123, A132), regarda (A423). L'absence de PS à l'écrit en $2^{\text {ème }}$ provient peut-être des conditions moins formelles de recueil des données. Les données de $1^{\text {ère }}$ avaient été relevées durant la semaine d'accueil à l'université et on peut supposer que les étudiants étaient soucieux de bien faire, l'enquête en $4{ }^{\text {ème }}$ se déroulait pendant le cours de langue écrite. Par contre, en deuxième année, les données ont été obtenues par des étudiants auprès de collègues volontaires et en l'absence du chercheur.

Pour ce qui est des PS à l'oral en $2^{\text {ème }}$, ils sont produits par deux apprenants A202 (fit, épousa) et A213 (sortit). Dans le cas du second informant, le cotexte semble indiquer que sortit est un présent d'intention :

(5) il est minuit moins quart et elle souvient que la marraine dit que devient-devient euh qu'elle a besoin revient avant minuit donc elle sortit du bal et elle quitte le prince retourne a sa maison

L'approche purement quantitative révèle des différences marquantes dans l'emploi du PS par les francophones d'une part et les apprenants de l'autre. Elle montre aussi l'influence d'une exposition extra-académique au français dans l'utilisation de la forme car le groupe des apprenants bilingues présente un pourcentage d'emploi du PS nettement plus élevé que celui des apprenants en milieu académique. On pourrait se demander quel facteur est le plus propice à l'utilisation du PS chez les bilingues : l'influence familiale ou le séjour à l'étranger. Le tableau ci-dessous résume l'arrière-plan de nos huit bilingues et leur production du PS dans la narration écrite, la narration orale et la liste de dix formes :

${ }^{17}$ Le PS constitue plus de la moitié des tiroirs utilisés (de 51,92\% à 81,36\%) pour tous les locuteurs natifs sauf N09 (33,33\%) qui offre le plus court des récits.

${ }^{18}$ L'emploi du PS est moins généralisé qu'à l'écrit : quatre des locuteurs natifs (N01, N07, N09 et N11) ne l'utilisent pas et les autres l'utilisent de façon moins systématique, allant d'une occurrence (1,96\%) chez N12 à 64\% chez N05. 


\begin{tabular}{|c|c|c|c|c|c|}
\hline & $\begin{array}{l}\text { Influence } \\
\text { familiale }\end{array}$ & $\begin{array}{ll}\text { Séjour à } \\
\text { l'étranger }\end{array}$ & $\begin{array}{l}\text { Nombre de } \\
\text { PS à l'écrit }\end{array}$ & $\begin{array}{l}\text { Nombre de PS } \\
\text { à l'oral }\end{array}$ & $\begin{array}{l}\text { Nombre } \\
\text { de PS } \\
\text { exact } \\
\text { dans le } \\
\text { test }\end{array}$ \\
\hline B01 & $\begin{array}{l}\text { Famille } \\
\text { bilingue }\end{array}$ & $\begin{array}{l}3 \text { ans en } \\
\text { Belgique }\end{array}$ & $1^{19}$ & $\varnothing$ & $2^{20}$ \\
\hline B02 & $\begin{array}{l}\text { Famille } \\
\text { bilingue }\end{array}$ & $\begin{array}{l}14 \text { ans en } \\
\text { France }\end{array}$ & $15^{21}$ & $57^{22}$ & $9^{23}$ \\
\hline B03 & & $\begin{array}{l}7 \text { ans en } \\
\text { France }\end{array}$ & $7^{24}$ & $\varnothing$ & $9^{25}$ \\
\hline B04 & & $\begin{array}{l}8 \text { ans en } \\
\text { France }\end{array}$ & $17^{26}$ & $9^{27}$ & $10^{28}$ \\
\hline B05 & & $\begin{array}{l}17 \text { ans à l'île } \\
\text { Maurice }\end{array}$ & $5^{29}$ & $\varnothing$ & $7^{30}$ \\
\hline B06 & & $\begin{array}{l}12 \text { ans en } \\
\text { France }\end{array}$ & $15^{31}$ & $\varnothing$ & $9^{32}$ \\
\hline B07 & & $\begin{array}{l}5 \text { ans en } \\
\text { France }\end{array}$ & $\varnothing$ & $\varnothing$ & $\varnothing$ \\
\hline B08 & Famille & & $2^{33}$ & $1^{34}$ & $\varnothing$ \\
\hline
\end{tabular}

19 Vécurent.

20 Chanta, donna.

21 Apparut, cessèrent, commença, demanda (2), donna, obéit, ordonna, put, se cacha, se remaria, se trouva, transforma (2), vint.

22 Accourut, aida, alla (3), arrêtèrent, arriva, attrista, cessèrent (2), commencèrent (2), courut, demanda (2), disparut, donna, durent, entendit, entra, eurent (2), fut, furent (2), garda, invita, mit, obéit (2), ordonna, ouvrit, partirent, passa, perdit, prit, ramena (2), rapporta, reconnurent, regarda, s'enfuit (2), se marièrent, se mit (2), se transformèrent, se trouva, se tut, tapa, transforma (3), vida, vit (2), *vivrèrent

23 fut, chanta, sentit, put, finit, donna, rendit + *dît et *sût.

24 Appela (2), décida, dit, se mit, se mirent, trouva.

25 Chanta, sentit, dit, finit, donna, rendit + *fût, *fit, *sût.

26 *Apparu, disparu, épousa, fut, furent, invita (2), *parti, proposa, *recevèrent, salua, se passa, sonna (2), vit.

27 Arriva (3), dit, fut, fit, partit, *réponda, répondit.

28 Fut, chanta, sentit, put, fit, dit, finit, donna, rendit et *sû.

29 Demanda, demandèrent, donna, pardonna, quitta.

30 Chanta, dit, sut, finit, donna + fut pour faire et *senta.

31 Changea (2), *courra, dansa, *devenna, *disa, donna, passa (2), *perda, *prenna (2), prépara, ramena.

32 Chanta, sut, donna + *senti et *pu +*faissa, *disa, *finissa, *renda.

33 Eurent, vécurent.

34 Interdit 


\begin{tabular}{|l|l|l|l|l|}
\hline & bilingue & & & \\
\hline \multicolumn{5}{c|}{ Tableau 3 : Arrière-plan personnel des sujets bilingues }
\end{tabular}

Nos informations personnelles ne permettent pas de trancher définitivement sur l'influence respective du milieu familial et du séjour dans un pays francophone. D'une part, les trois sujets issus de familles franco-anglaises possèdent à des degrés divers le PS : on ne dispose pas des informations permettant d'évaluer la place réelle du français dans ces familles. Il semble d'autre part que plus les sujets ont séjourné dans un pays francophone, plus ils maîtrisent le PS, sauf B07 qui, quoique natif à l'oral, semble ignorer la forme. Il se pourrait que l'environnement scolaire soit ici décisif et que la maîtrise du PS dépende de la fréquentation d’une école francophone à l’âge où le PS est enseigné. Nos données biographiques limitées ne nous permettent malheureusement pas de vérifier cette hypothèse de façon satisfaisante.

Concentrons-nous maintenant sur la production métalinguistique du PS dans la liste de dix formes verbales soumises à nos sujets. La production de ces formes hors contexte a révélé deux points intéressants. D’une part, N03 et N06 ont systématiquement fourni des PC. Bien qu'étant nés en France et ayant effectué toute leur scolarité en milieu francophone, ces deux sujets sont des immigrés d’Extrême-Orient de deuxième génération et auraient pu ne pas bénéficier du même input familial que les autres locuteurs natifs, Toutefois, N03 produit 19 PS à l'écrit et 17 à l'oral tandis que N06 en utilise 25 à l'écrit et 10 à l'oral, la forme est donc clairement productive dans leur idiolecte. Leur erreur métalinguistique provient sans doute de leur formation académique : ces deux sujets sont des doctorants en informatique alors que les autres francophones proviennent d'un cursus en sciences du langage. D'autre part, les francophones rencontrent des problèmes morphologiques avec le PS. Il peut s'agir (1) de PS' = formes au PS mais inexactes (ex. : fit pour être), (2) de PS'” = formes mal orthographiées (ex. : senti pour sentit), (3) de PS'” = formes inventées à désinence de PS (ex. : *pouva).

\begin{tabular}{|l|l|l|l|l|l|l|l|l|l|l|}
\hline & Etre & chanter & sentir & pouvoir & faire & dire & savoir & finir & donner & rendre \\
\hline PS & 66,67 & 77,78 & 66,67 & 66,67 & 55,56 & 77,78 & 55,56 & 55,56 & 77,78 & 66,67 \\
\hline PS' & & & & & 11,11 & & & & & \\
\hline PS', & 11,11 & & & 11,11 & 11,11 & & 22,22 & 22,22 & & \\
\hline PS',' & & & & & & & & & & 11,11 \\
\hline PC & 22,22 & 22,22 & 22,22 & 22,22 & 22,22 & 22,22 & 22,22 & 22,22 & 22,22 & 22,22 \\
\hline Pres & & & 11,11 & & & & & & & \\
\hline
\end{tabular}

Tableau 4 : Distribution des formes produites comme PS par les locuteurs natifs

Comparés aux francophones, les locuteurs bilingues produisent un éventail plus large de formes avec une confusion des temps du passé, la création de formes et des abstentions. On pourrait peut-être attribuer cette compétence 
inférieure au déficit académique, ces sujets n’ayant pas nécessairement suivi des cours de français, langue maternelle.

\begin{tabular}{|l|l|l|l|l|l|l|l|l|l|l|}
\hline & Etre & chanter & sentir & pouvoir & faire & dire & savoir & finir & donner & rendre \\
\hline PS & 20 & 60 & 30 & 20 & 10 & 30 & 20 & 40 & 60 & 30 \\
\hline PS' & & & & & 20 & & & & & \\
\hline PS'’ & 10 & & 10 & 20 & 10 & 10 & 30 & & & \\
\hline PS'”, & & & 20 & 10 & 20 & 20 & 10 & 20 & & 30 \\
\hline PC & 10 & 10 & 10 & 20 & 10 & 10 & 20 & 20 & 10 & 10 \\
\hline IMP & 30 & 20 & 20 & 10 & 20 & 20 & 10 & 10 & 20 & 10 \\
\hline SUBJ & 10 & & & & & & & & & \\
\hline PP & 10 & 10 & & & & & & & 10 & \\
\hline cond & & & & & & & & & & 10 \\
\hline$?$ & & & 10 & 10 & 10 & 10 & 10 & 10 & & 10 \\
\hline$\varnothing$ & 10 & & & 10 & & & & & & \\
\hline
\end{tabular}

Tableau 5 : Distribution des formes produites comme PS par les bilingues

On constate chez les apprenants de $4^{\text {ème }}$ et de $2^{\text {ème }}$ années un taux d'abstention important ainsi qu'une confusion avec le PC en $4^{\text {ème }}$ année, l'absence du problème en $2^{\text {ème }}$ pourrait venir de la méthode de rassemblement de données en face à face qui permettait au sujet de s'assurer plus facilement de ce qu'était le PS :

\begin{tabular}{|l|l|l|l|l|l|l|l|l|l|l|}
\hline & Etre & chanter & sentir & pouvoir & faire & dire & savoir & finir & donner & rendre \\
\hline PS & 30,77 & & 7,69 & & 7,69 & 7,69 & & & 15,38 & \\
\hline PS' & 7,69 & 15,38 & & & 7,69 & & & & & \\
\hline PS', & 7,69 & & 7,69 & 15,38 & & 7,69 & 7,69 & 15,38 & & \\
\hline PS', & & & & & & & 7,69 & & & 15,38 \\
\hline PC & 23,08 & 30,77 & 15,38 & 15,38 & 23,08 & 15,38 & 15,38 & 15,38 & 15,38 & 15,38 \\
\hline$\varnothing$ & 23,08 & 53,85 & 69,23 & 69,23 & 61,54 & 69,23 & 69,23 & 69,23 & 69,23 & 69,23 \\
\hline$?$ & 7,69 & & & & & & & & & \\
\hline
\end{tabular}

Tableau 6 : Distribution des formes produites comme PS par les $4^{\text {ème }}$ année

\begin{tabular}{|l|l|l|l|l|l|l|l|l|l|l|}
\hline & Etre & chanter & sentir & pouvoir & faire & dire & savoir & finir & donner & rendre \\
\hline PS & 46,15 & 53,85 & 15,38 & 15,38 & 15,38 & 7,69 & 15,38 & 15,38 & 53,85 & 7,69 \\
\hline PS' & 7,69 & & & & 15,38 & & & & & \\
\hline PS'’ & & & 7,69 & 7,69 & 7,69 & & 7,69 & & & \\
\hline PS’’’ & & & 7,69 & 7,69 & 7,69 & 15,38 & 7,69 & 7,69 & & \\
\hline Pres & & 7,69 & 7,69 & & & & & & 7,69 & 7,69 \\
\hline$\varnothing$ & 46,15 & 30,77 & 53,85 & 61,54 & 53,85 & 69,23 & 69,23 & 69,23 & 38,46 & 76,92 \\
\hline PP & & & & & & & & & & 7,69 \\
\hline$?$ & & 7,69 & 7,69 & 7,69 & & 7,69 & & 7,69 & & \\
\hline
\end{tabular}

Tableau 7 : Distribution des formes produites comme PS par les $2^{\text {ème }}$ année 
Les résultats de première année révèlent une confusion à la fois terminologique et morphologique. Les sujets semblent incertains de ce qu'est le PS et produisent des formes inventées.

\begin{tabular}{|l|l|l|l|l|l|l|l|l|l|l|}
\hline & Etre & chanter & sentir & pouvoir & faire & dire & savoir & finir & donner & rendre \\
\hline PS & 5,88 & 5,88 & 11,76 & 5,88 & & 17,65 & 5,88 & 17,65 & 2,94 & \\
\hline PS' & 8,82 & 2,94 & & & 17,65 & 2,94 & & & & \\
\hline PS'’ & 2,94 & & 8,82 & 2,94 & & & 5,88 & 2,94 & & \\
\hline PS',' & & & & 8,82 & & 2,94 & & & 2,94 & 5,88 \\
\hline PC & 17,65 & 17,65 & 17,65 & 14,71 & 8 & 17,65 & 14,71 & 17,65 & 17,65 & 17,65 \\
\hline IMP & 14,71 & 8,82 & 8,82 & 2,94 & 11,76 & 8,82 & 8,82 & 8,82 & 8,82 & 8,82 \\
\hline COND & & & & 2,94 & & & & & & \\
\hline PRES & & 5,88 & & 2,94 & 5,88 & & 5,88 & & 5,88 & \\
\hline PQP & & 2,94 & 2,94 & 2,94 & & 2,94 & 2,94 & 2,94 & 2,94 & 2,94 \\
\hline SUBJ & & & & & & & & 2,94 & & 2,94 \\
\hline$\varnothing$ & 38,24 & 44,12 & 44,12 & 50 & 41,18 & 44,12 & 50 & 47,06 & 50 & 50 \\
\hline PP & 5,88 & 8,82 & & & & 2,94 & & & 8,82 & 8,82 \\
\hline$?$ & 5,88 & 2,94 & 5,88 & 5,88 & & & 5,88 & & & 2,94 \\
\hline
\end{tabular}

Tableau 8 : Distribution des formes produites comme PS par les $1^{\text {ère }}$ année

Les données quantitatives étudiées dans cette section soulignent plusieurs caractéristiques du corpus. Ainsi, les apprenants ne recourent qu'occasionnellement au PS dans leur production spontanée écrite et orale. Le PS apparaît plus fréquemment dans la production métalinguistique et la proportion de formations correctes (PS) et d'essais (PS', PS',, PS',') augmente parallèlement au niveau d'avancement linguistique.

\begin{tabular}{|l|l|l|l|l|l|}
\hline & Francophones & Bilingues & $4^{\text {ème }}$ année & $2^{\text {ème }}$ année & $1^{\text {ère } \text { année }}$ \\
\hline PS & 60,00 & 32 & 6,92 & 23,08 & 7,35 \\
\hline PS' & 1,11 & 2 & 9,99 & 2,31 & 3,23 \\
\hline PS'” & 7,70 & 9 & 6,15 & 3,08 & 2,65 \\
\hline PS'”, & 1,11 & 13 & 2,31 & 5,38 & 2,06 \\
\hline & 69,92 & 56 & 25,37 & 33,85 & 14,99 \\
\hline
\end{tabular}

Tableau 9 : Pourcentages des formes du PS ou assimilées

Si les francophones produisent régulièrement le PS dans leurs narrations et sont en général capables de former ce tiroir, leur maîtrise n’en est pas moins imparfaite et des problèmes morphologiques et surtout orthographiques subsistent; ceux-ci suggèrent que le PS ne fait pas partie du répertoire courant de nos sujets.

\subsection{Influence de l’input}

Krashen $(1985,2)$ a avancé l' « Input Hypothesis » selon laquelle « [...] humans acquire language in only one way - by understanding messages, or 
by receiving 'comprehensible input' ». Les apprenants sont capables de comprendre des extraits langagiers comprenant des éléments de grammaire non acquis grâce au contexte, à la connaissance du monde et à la compétence linguistique préalable. L'instruction formelle peut jouer un rôle facilitateur en ce qu'elle simplifie l'input trop complexe.

Pour tenter d'évaluer une possible influence de l'input sur l'utilisation du PS, on a sélectionné différentes catégories d'apprenants : certains n’ayant pas eu d'enseignement explicite en faculté (première année), d'autres en ayant reçu un (deuxième année). En outre, les sujets de quatrième ont bénéficié d'une immersion en pays francophone. On s'attendrait à ce que les étudiants de première année fassent preuve d'une performance moindre.

De plus, pour évaluer l'effet de l'input sur la productivité, on avait séparé chacun des groupes en deux : la première moitié lisait le conte en version anglaise (A101 à A 116; A201 à A207; A401 à A408; B01 à B04 ; N01 à N07)) et la seconde en version française (A117 à A134; A208 à A213; A409 à A416; B05 à B08; N08 à N13) . On aurait attendu une proportion plus élevée de PS dans le deuxième groupe.

\begin{tabular}{|l|l|l|l|l|l|l|l|l|}
\hline & \multicolumn{4}{|l|}{ Version anglaise } & \multicolumn{4}{l|}{ Version française } \\
\hline & \multicolumn{2}{|l|}{ Écrit } & \multicolumn{2}{l|}{ Oral } & \multicolumn{2}{l|}{ Écrit } & \multicolumn{2}{l|}{ Oral } \\
\hline $1^{\text {ère }}$ année & 11 & 2,12 & 0 & 0 & 12 & 1,75 & 0 & 0 \\
\hline $2^{\text {ème }}$ année & 0 & 0 & 2 & 0,61 & 0 & 0 & 1 & 0,42 \\
\hline $4^{\text {ème }}$ année & 4 & 1,43 & 2 & 0,45 & 3 & 1,02 & 0 & 0 \\
\hline Bilingues & 42 & 28 & 10 & 3,21 & 23 & 17,69 & 1 & 0,28 \\
\hline $\begin{array}{l}\text { Locuteurs } \\
\text { natifs }\end{array}$ & 188 & 63,73 & 108 & 26,02 & 141 & 59,24 & 87 & 24,37 \\
\hline
\end{tabular}

Tableau 10 : Proportion relative du PS dans les narrations basées sur les scripts anglais et français du conte

Les résultats vont à l'encontre des attentes puisque les sujets ayant lu la version anglaise recourent plus au PS que ceux qui se sont basés sur le texte français. La présence du PS dans l'input ne semble donc pas ici favoriser l'emploi de cette forme.

Il faut toutefois noter que les deux formes vécurent et eurent mises par écrit et laissées sous les yeux des sujets (contrairement au texte lui-même) ont été utilisées par les première année. Il se pourrait simplement que le PS ne se manifeste que s'il est déjà bien implanté dans l'IL des apprenants et une simple lecture à but informationnel ne suffit pas pour repérer les formes verbales caractéristiques du type de texte.

\subsection{Utilisation productive ou formulaire?}


Ayant constaté la présence occasionnelle du PS à tous les niveaux d'apprentissage, nous allons nous interroger sur la productivité de la forme dans l'interlangue avancée. Une analyse qualitative des PS révèle un emploi largement formulaire chez les apprenants: à l'écrit, 16 emplois sur 23 $(69,57 \%)$ en $1^{\text {ère }}$ et 5 sur $8(62,5 \%)$ en $4^{\text {ème }}$ sont des reprises de ils furent heureux et eurent beaucoup d'enfants. Les autres formes sont majoritairement monosyllabiques : dit (A132), fut (A106), deux fit (A127), se mit (A128, A407). Dans le groupe bilingue, l'éventail de verbes au PS est nettement plus large ${ }^{35}$ et on peut difficilement soutenir l'hypothèse de " chunk learning » étant donné que des formes non natives régulières sont produites, particulièrement par B07 qui produit courra, devenna et perda.

\subsection{Genre contre medium}

On ne s'étendra pas sur l'affinité du PS avec l'écrit ; elle a été largement documentée ailleurs. On sait par ailleurs que le PS est un ressort déterminant du conte de fées traditionnel ${ }^{36}$. Compte tenu de ces tendances contradictoires, on voulait voir si une reddition orale allait invalider l'emploi du PS. Si l'on constate que le PS est, à l'exception de la $2^{\text {ème }}$ année, moins utilisé à l'oral qu'à l'écrit, l'emploi du PS est trop limité dans les corpus d'apprenants pour en tirer des conclusions très convaincantes. Cependant, le corpus natif montre que dans le genre du conte, le PS s'utilise à l'oral en concurrence avec le PRES et pas le PC.

\subsection{Facteurs favorisant l'utilisation du PS}

Dans cette section, nous allons tenter d'identifier des facteurs propices à l'utilisation du PS. Nous nous pencherons d'abord sur des indices contextuels comme le genre narratif avant d'aborder des éléments cotextuels tels que la structure narrative du récit.

35 Appela (2) (B04), apparut (B03, B05), eurent (B02, B09), cessèrent (B03), changea (2), (B07), commença (B03), courra (B07), dansa (B07), décida (B04), demanda (B02, B03 [2], B06), demandèrent (B06), devenna (B07), dit (B04), donna (B03, B06, B07), épousa (B05), fut (B05), furent (B05), invita (B05), abéit (B03), ordonna(B03), pardonna (B06), partit (B05), passèrent (2) (B07), perda (B07), put (B03), prenna (2) (B07), prépara (B07), proposa (B05), quitta (B06), ramena (B07), recevèrent (B05), salua (B05), se cacha (B03), se mit (B04), se mirent (B04), se passa (B05), se remaria (B03), se trouva (B03), sonna (2) (B05), transforma (2)(B03), trouva (B04), vint (B03), vida (B03), vit (B05), vécurent (B01, B02, B09) à l'écrit.

${ }^{36}$ Les premiers résultats de la recherche de Janice Carruthers sur le «néo-conte » indiquent que cette contrainte n'y est pas essentielle. 


\subsubsection{Influence du contexte}

On constate que les francophones narrent le conte de fées différemment d'autres récits, tels que des extraits de films (Labeau 2002 et 2005). Alors que les péripéties du film se racontent à l'écrit au PC et, à l'oral au PC ou au PRES $^{37}$, le conte de fées s'écrit au PS et se dit principalement au PS et au PRES. Il existe donc une forte influence du genre sur le choix des marqueurs verbaux.

\begin{tabular}{|l|l|l|l|l|}
\hline & \multicolumn{3}{|l|}{ Film } & \multicolumn{2}{l|}{ Conte } \\
\hline & Écrit & Oral & Écrit & Oral \\
\hline PRES & 8 & 33.21 & 0.38 & 19.4 \\
\hline PS & 10.5 & 0 & 61.73 & 25.26 \\
\hline PA & & & 0.19 & 0.26 \\
\hline PC & 59.5 & 47.71 & 0.38 & 6.48 \\
\hline IMP & 14 & 11.93 & 27.77 & 29.79 \\
\hline PQP & 6.5 & 5.05 & 3.19 & 3.37 \\
\hline FPP & & & & 0.26 \\
\hline FS & 0 & 0.18 & 0 & 0.13 \\
\hline FP & 0 & 0 & 0 & 0.91 \\
\hline COND & 0 & 0.92 & 3.19 & 2.46 \\
\hline CP & & 0.18 & 0.75 & 0.13 \\
\hline SUBJ & 1 & 0.36 & 2.06 & 1.17 \\
\hline $\begin{array}{l}\text { SUBJ } \\
\text { P }\end{array}$ & 0.5 & & 0.38 & \\
\hline IMPER & & & & 0.39 \\
\hline
\end{tabular}

Tableau 11: Pourcentage d'emplois des temps dans la narration d'un film et d'un conte par des locuteurs natifs

Si l'on compare maintenant l'emploi des apprenants dans la narration des deux genres, on constate une légère hausse de l'utilisation du PS, qui reste un marqueur marginal. Les traits les plus remarquables sont d'une part la fréquence du PRES, théoriquement exclu par la consigne de raconter au passé lors du recueil des narrations de films, qui augmente considérablement sauf en $4^{\text {ème }}$, et d'autre part, la fréquence plus élevée de l'IMP à tous les niveaux due à la plus grande composante de description dans le conte et la longueur des narrations dans le corpus de $4{ }^{\text {ème }}$ qui laisse plus de place aux informations d'arrière-plan à l'IMP.

\footnotetext{
${ }^{37}$ La tendance naturelle est de raconter au PRES (Salaberry 2000) mais lors de la récolte du corpus basé sur des extraits de film, les locuteurs natifs avaient reçu l’instruction de raconter au passé.
} 


\begin{tabular}{|c|c|c|c|c|c|c|c|c|c|c|c|c|}
\hline & \multicolumn{6}{|l|}{ Film } & \multicolumn{6}{|l|}{ Conte } \\
\hline & \multicolumn{3}{|l|}{ Écrit } & \multicolumn{3}{|l|}{ Oral } & \multicolumn{3}{|l|}{ Écrit } & \multicolumn{3}{|l|}{ Oral } \\
\hline & $\begin{array}{l}\text { 1ère } \\
\text { année }\end{array}$ & $\begin{array}{l}\text { 2ème } \\
\text { année }\end{array}$ & $\begin{array}{l}\text { 4ème } \\
\text { année }\end{array}$ & $\begin{array}{l}\text { 1ère } \\
\text { année }\end{array}$ & $\begin{array}{l}\text { 2ème } \\
\text { année }\end{array}$ & $\begin{array}{l}\text { 4ème } \\
\text { année }\end{array}$ & $\begin{array}{l}\text { lère } \\
\text { année }\end{array}$ & $\begin{array}{l}\text { 2ème } \\
\text { année }\end{array}$ & $\begin{array}{l}\text { 4ème } \\
\text { année }\end{array}$ & $\begin{array}{l}\text { 1ère } \\
\text { année }\end{array}$ & $\begin{array}{l}\text { 2ème } \\
\text { année }\end{array}$ & $\begin{array}{l}\text { 4ème } \\
\text { année }\end{array}$ \\
\hline PRES & 5,35 & 6,52 & 3,42 & 26,19 & 9,33 & 23,52 & 18,12 & 31,63 & 10,82 & 31,89 & 28,55 & 10,99 \\
\hline PS & 0,16 & 0 & 1,5 & 0,58 & 0 & 0 & 1,91 & 0 & 1,22 & 0 & 0,53 & 0,23 \\
\hline PC & 58,49 & 68,56 & 66,12 & 56,34 & 63,56 & 53,83 & 33,42 & 31,63 & 36,3 & 32,65 & 34,04 & 41,68 \\
\hline PSC & & & & & & & & & & 0,07 & & \\
\hline IMP & 31,92 & 20,4 & 25,68 & 15,25 & 23,59 & 20,33 & 32,5 & 28,6 & 43,98 & 28,26 & 29,26 & 37,83 \\
\hline FPP & & & & & & & & 0,23 & 0,35 & 0,07 & & 0,68 \\
\hline PQP & 1,73 & 2,55 & 1,64 & 0,35 & 2,19 & 0,56 & 2,08 & 0,93 & 10,82 & 0,89 & 1,42 & 3,49 \\
\hline FS & 0 & 0,28 & 0,14 & 0 & 0 & 0,16 & 2,83 & 0,47 & 0,35 & 0,14 & 0,35 & 0,34 \\
\hline FP & 0 & 0,28 & 0,27 & 0,23 & 0,73 & 0,4 & 0,42 & 1,4 & 0,87 & 0,34 & 1,06 & 1,25 \\
\hline COND & 0,63 & 0,85 & 0,14 & 0,12 & 0 & 0,4 & 2,83 & 2,09 & 0,87 & 0,19 & 1,77 & 1,25 \\
\hline $\mathrm{CP}$ & & & & & 0,15 & 0 & & & & 0,07 & 0,18 & 0,23 \\
\hline SUBJ & 0,31 & 0 & 0,27 & 0,12 & 0,29 & 0,48 & 0,83 & 0,23 & 1,57 & 0,75 & 0,35 & 1,59 \\
\hline $\begin{array}{l}\text { SUBJ } \\
\mathrm{P}\end{array}$ & 0 & 0 & 0,27 & & & & 0 & & & & & \\
\hline $\begin{array}{l}\text { SUBJ } \\
\text { IMP }\end{array}$ & & & & & & & & & 0,17 & & & 0,11 \\
\hline IMPER & & & & 0,12 & 0 & 0,16 & 0,15 & & 0,17 & 0,82 & 0,53 & 0,34 \\
\hline ? & 1,42 & 0,57 & 0,55 & 0,7 & 0,58 & 0,16 & 6,82 & 2,79 & 2,27 & 1,71 & 1,95 & 1,02 \\
\hline
\end{tabular}

Tableau 12 : Pourcentage d'emplois des temps dans la narration d'un film et d'un conte par des apprenants anglophones

On constate donc que la dimension stylistique de la narration échappe aux apprenants, qui semblent ignorer l'équation 'contes de fées = PS' que manient les francophones au moins à l'écrit. Le corpus formé de narrations de films ne comprenait pas de groupe bilingue, mais il est probable que la proportion de PS y aurait été beaucoup plus limitée que dans le corpus de contes de fées.

\subsubsection{Influence du cotexte (la position dans le texte)}

On va maintenant s'interroger sur l'influence possible de la structure narrative dans l'utilisation du PS. Cette forme apparaîtrait-elle préférentiellement à certains points du récit?

Propp a proposé une structure du conte en 31 points qui peuvent se regrouper en 4 catégories : (1) la séquence d’introduction, (2) le corps du texte, (3) la séquence du don (obtention d'une aide magique) et (4) retour du héros. Dans la version de Cendrillon étudiée, on a relevé les points suivants :

\section{(1) Séquence d'introduction}

1. Un membre de la famille disparaît (la mère) et l'héroïne est présentée. 
2. L'interdiction: Cendrillon doit renoncer à ses prérogatives de fille de la maison.

7. La soumission : Cendrillon accepte son sort sans se plaindre.

(2) Corps du texte

8. La vilenie : la belle-mère et les belles-sœurs de Cendrillon la privent de bal tout en la tentant.

9. Le manque : Cendrillon révèle à sa marraine son désir d'assister au bal.

10. L'accord à l'action : Cendrillon se soumet aux ordres de sa marraine.

(3) La séquence du don

12. Le test : la fée teste la motivation de Cendrillon en lui donnant des ordres apparemment ridicules.

13. La réaction : Cendrillon obéit.

14. L'objet magique : Cendrillon reçoit par magie les moyens d'aller au bal.

15. Le transfert vers le lieu où le manque va être comblé : Cendrillon part pour le bal.

19. Disparition du manque : Cendrillon est honorée.

(4) Le retour du héros

20. Le départ : Cendrillon quitte le bal [ retour en arrière].

18. La défaite du méchant : les méchantes sœurs doivent s'incliner devant la beauté de l'inconnue.

19. Disparition du manque : Cendrillon est honorée.

20. Le départ : Cendrillon quitte le bal.

21. La poursuite : le prince tente de la rattraper.

22. L'échappée : Cendrillon disparaît en abandonnant sa pantoufle.

23. Le retour sans reconnaissance : Cendrillon reprend sa place de souillon.

24. Les faux héros : les méchantes sœurs tentent d'enfiler la pantoufle.

25. L'épreuve: Cendrillon demande à essayer la pantoufle sous les moqueries.

26. La victoire : La pantoufle sied à Cendrillon.

27. La reconnaissance: Cendrillon confirme son identité par la seconde pantoufle.

28. Les méchants démasqués : les méchantes sœurs s’humilient.

29. L'épiphanie : Cendrillon est menée au prince dans un habit de lumière.

31. Le mariage et le règne du héros.

Certaines des catégories évoquent la succession des péripéties, alors que d'autres (principalement la séquence d'introduction et les états résultants final $(31)$ ou intermédiaires $(19,23)$ sont propices à la description. Ces contrastes sont susceptibles de se manifester dans la morphologie verbale : le PS se prêterait à l'expression des événements successifs (voir Bres 2003, Vetters 2003) alors que l'IMP conviendrait mieux aux parties descriptives. 
Le tableau suivant explicite la répartition proportionnelle des PS dans les narrations écrites ${ }^{38}$ pour les différentes catégories relevées. Il montre des différences majeures entre les natifs et les bilingues d'une part, et les apprenants de l'autre, dans l'emploi discursif du PS.

\begin{tabular}{|c|c|c|c|c|c|c|c|c|}
\hline \multirow[b]{2}{*}{ Catégories } & \multicolumn{2}{|c|}{ Corpus natif } & \multicolumn{2}{|c|}{$1^{\text {ère }}$ année } & \multicolumn{2}{|c|}{$4^{\text {ème }}$ année } & \multicolumn{2}{|c|}{ Bilingues } \\
\hline & $\begin{array}{l}\mathrm{Nb} \\
\mathrm{PS}\end{array}$ & $\%$ & $\begin{array}{l}\mathrm{Nb} \\
\mathrm{PS}\end{array}$ & $\%$ & $\begin{array}{l}\mathrm{Nb} \\
\mathrm{PS}\end{array}$ & $\%$ & $\begin{array}{l}\mathrm{Nb} \\
\mathrm{PS}\end{array}$ & $\%$ \\
\hline 1 & 14 & 4,26 & & & 1 & 14,29 & 2 & 3,23 \\
\hline 2 & 11 & 3,34 & 2 & 8,7 & & & 6 & 9,68 \\
\hline 7 & 3 & 0,91 & & & & & 1 & 1,61 \\
\hline 8 & 38 & 11,55 & 2 & 8,7 & & & 9 & 15,42 \\
\hline 9 & 29 & 8,81 & 1 & 4,35 & 1 & 14,29 & 4 & 6,45 \\
\hline 10 & 3 & 0,91 & & & & & & \\
\hline 12 & 8 & 2,43 & & & & & 3 & 4,84 \\
\hline 13 & 3 & 0,91 & & & & & 1 & 1,61 \\
\hline 14 & 41 & 12,46 & & & & & 12 & 19,35 \\
\hline 15 & 9 & 2,74 & & & & & 2 & 3,23 \\
\hline 19 & 26 & 7,9 & & & & & 7 & 11,29 \\
\hline 20 & 11 & 3,34 & & & & & 4 & 6,45 \\
\hline 18 & 7 & 2,13 & & & & & & \\
\hline 19 & 22 & 6,69 & & & & & 2 & 3,23 \\
\hline 20 & 10 & 3,04 & 2 & 8,7 & & & 1 & 1,61 \\
\hline 21 & 1 & 0,3 & & & & & & \\
\hline 22 & 9 & 2,74 & & & & & 3 & 4,84 \\
\hline 23 & 18 & 5,47 & & & & & & \\
\hline 24 & 9 & 2,74 & & & & & & \\
\hline 25 & 13 & 3,95 & & & & & & \\
\hline 26 & 7 & 2,13 & & & & & & \\
\hline 27 & 5 & 1,52 & & & & & & \\
\hline 28 & 2 & 0,61 & & & & & 2 & 3,23 \\
\hline 29 & 5 & 1,52 & & & & & & \\
\hline \multirow[t]{2}{*}{31} & 25 & 7,6 & 16 & 69,57 & 5 & 71,43 & 3 & 4,84 \\
\hline & 329 & 100 & 23 & 100 & 7 & 100 & 62 & 100 \\
\hline
\end{tabular}

Les francophones et les bilingues utilisent le PS comme ressource narrative constante: on relève des occurrences dans toutes (ou la plupart de) les catégories; la fréquence du PS est, comme on pouvait s'y attendre, plus importante aux points-clés de l'histoire : l'invitation (8), la transformation (14), le bal (19) et la conclusion (31). Chez les apprenants, les occurrences du

${ }^{38}$ Nous nous limitons ici aux narrations écrites qui comprennent une plus grande fréquence de PS. Des phénomènes comparables sont aussi perceptibles dans les narrations orales. 
PS sont isolées et apparemment aléatoires, sauf pour (31) mais on a vu plus haut que ces emplois étaient formulaires. Dans les corpus d'apprenants, l'absence du PS est palliée par d'autres formes verbales : le PC bien entendu mais aussi des IMP non natifs et des PRES :

(6) Quand les soeurs ont quitté la maison, la marenne de Cendrillon a apparu. Cendrillon pleurait. " Est-ce que tu veut aller au bal ", la marenne a demandé ? «Bien sur ! " Cendrillon a répondu. "Bon, trouve-moi un légume, 6 souris, 1 rat et 3 lizards ». Cendrillon a trouvé tous ses choses dans le jardin et elle a donné ces choses à sa marenne. La marenne a fait la magie. (A414)

(7) Cendrillon était très triste parce qu'elle ne pouvait pas y aller. Cendrillon décidait de demander sa marraine si elle pouvait aller au bal, la marraine disait qu'il faut que Cendrillon cherche une légume dans le jardin. Cendrillon trouvait la légume, la marraine utilisait son pouvoir et dans un flashe Cendrillon portait des beaux vêtements et il $\mathbf{y}$ avait des chevaux, qui étaient prêts de la prendre au bal. Avant que Cendrillon partitit la marraine la disait qu'elle devait rentrer chez elle avant minuit. (A412)

(8) Pendant la soirée du ball, Cinderellion était faire la menage de la maison quand une femme gentile arrivait à sa maison. Cette femme était magicale et elle aidait Cinderellion à faire du ball. Elle changait des mices aux chevaux, et donnait Cinderellion un robe magnifique. Cinderellion arrivait du ball mais elle avait faire un promis de rentrer chez soi avant minuit. (A114)

(9) Les temps à arrivé pour la belle-mère et les soeurs d'allent à le bal et Cendrillon était triste quand une marriere arrivé. Cendrillon dit qu'elle sœurs sont horrible mais la femme aidé Cendrillon de prepare pour le bal. Elle change souris entre les chevaux et les rats entre trois hommes et une citroe entre un carrage. Elle aussi donne Cendrillon une belle robe et dit qu'elle doit retourné à minuit parce que les chevaux retourné à souris et les hommes à rats. (A126)

(10) Cendrillon reste a la maison. Dans un coup, sa *marriane apparaître et elle dit Cendrillon qu'elle doit aller au bal. Mais Cendrillon dit a sa marraine qu'elle n'est pas des vetements pour aller au bal.. la marraine dit Cendrillon d'amener 6 souris, et avec sa baguette elle transformer les souris à 6 chevaux. Elle demande Cendrillon d'amener aussi 3 rats et 6 lezards. La *marrine transformer les rats et les lezards au 3 hommes et 6 laquais. Enfin elle change les vetements de Cendrillon et elle devient une princesse. La *marriane dit Cendrillon de venir avant minuit car tout vas changer comme avant. (A213)

On constate que, contrairement aux francophones - et dans une certaine mesure aux bilingues -, les apprenants n’utilisent en règle générale pas le PS comme ressource narrative cohérente. Leurs emplois sont majoritairement isolés et semblent émerger de façon aléatoire. 


\section{Conclusion}

La présente étude nous permet de tirer quelques conclusions sur l'utilisation du PS par les apprenants avancés. D’abord, on constate que leur compétence dépasse leur performance dans ce domaine: si les PS sont rares en production spontanée, ils sont nettement plus nombreux en production sollicitée. En production spontanée, on constate une nette proportion de formes monosyllabiques (chunks ?) et de formes régulières ou régularisées. Quant au corpus bilingue, il nous a permis de constater des différences nettes entre leur appréhension du PS et celle des apprenants monolingues. La limitation de nos données ne nous a pas permis d'établir avec certitude le rôle respectif du séjour en pays francophone, de l'entourage familial ou de la scolarité en français dans l'acquisition de la forme. Compte tenu de l'utilisation limitée du PS par les $4{ }^{\text {ème }}$ année, on peut douter de l'influence du séjour. Il est plus difficile de trancher sur l'influence familiale (familiarisation avec les histoires à la française durant l'enfance) ou scolaire (enseignement formel du PS) ; la conscience du PS doublée du manque de maîtrise morphologique des sujets issus de familles bilingues semblerait indiquer que leur déficit par rapport aux francophones est d'ordre scolaire. Finalement, la présence limitée du PS dans les corpus d'apprenants souligne une fois de plus les limitations d'un modèle tel que l'hypothèse de l'aspect pour rendre compte de l'acquisition aspectuo-temporelle en français, langue étrangère. Il ne prend en effet pas plus en compte les variantes stylistiques que les variantes sémantiques (Labeau 2004b).

\section{Références}

Andersen R. (1986), 'El desarollo de la morphología verbal en el Español como segunda idioma', in Meisel J.M. (ed) Adquicisión de languaje / Acquisicao da linguagem, Frankfurt, Vervuert, 115-138.

Andersen R.W. (1991), 'Developmental sequences: the emergence of aspect marking in second language acquisition', in Huebner T. \& Ferguson, C.A. (eds), Crosscurrents in Second Language Acquisition Theories, Amsterdam / Philadelphia, John Benjamins.

Andersen R. (2002), 'The dimensions of “pastness”, in Salaberry R. \& Shirai Y., The L2 Acquisition of Tense-Aspect Morphology, Amsterdam / Philadelphia, John Benjamins, 79-105.

Andersen R. \& Shirai Y. (1994), 'Discourse motivations for some cognitive acquisition principles’, Studies in Second Language Acquisition 16, 133-156.

Bardovi-Harlig K. \& Bergström A. (1996), 'Acquisition of tense and aspect in second language and foreign language learning: learner narratives in ESL and FFL', Canadian Modern Language Review 52, 308-330.

Bartning I. (1997), 'L’apprenant dit avancé et son acquisition d'une langue étrangère. Tour d'horizon et esquisse d'une caractérisation de la variété avancée', in Bartning I. (dir.), Les apprenants avancés, AILE 9, 9-50. 
Bergström A. (1995), The expression of past temporal reference by English-speakers of French, Unpublished PhD dissertation, The Pennsylvania State University.

Gougenheim G., Rivenc P., Michéa R. \& Sauvageot A. (1964), L'élaboration du français fondamental, Paris, Didier.

Howard M. (2002), 'L'acquisition des temps du passé en français par l'apprenant dit avancé: une approche lexicale', in Labeau E. \& Larrivée P. (dir.), Les temps du passé et leur enseignement, Amsterdam-Atlanta, Rodopi, 181-204.

Judge A. \& Healey F.G. (1983), A Reference Grammar of Modern French, London, Ed. Arnold.

Krashen S.D. (1985), The Input Hypothesis : Issues and Implications, London and New York, Longman.

Labeau E. (2002), The Acquisition of French Past Tenses by Tutored Anglophone Advanced Learners: Is Aspect Enough?, unpublished PhD dissertation, Aston University.

Labeau E. (2004a), 'Line or circle? The process of past tenses acquisition by advanced learners of French', Proceedings of the 4th International Symposium on Bilingualism, Somerville, MA, Cascadilla Press.

Labeau E. (2004b), 'Au-delà de l'hypothèse de l'aspect : le développement du temps et de l'aspect dans l'interlangue d'apprenants avancés', Actes de la conférence APLIC, Paris 3, 25-26 juin 2004.

Labeau E. (2005), Beyond the Aspect Hypothesis: Tense-Aspect Development in advanced L2 French (Contemporary Studies in Descriptive Linguistics), Bern, Peter Lang.

Martin R. (1971), Temps et Aspect: Essai sur l'emploi des temps narratifs en moyen français, Paris, Klincksieck.

Pfister M. (1974), 'L’imparfait, le passé simple et le passé composé en français moderne', Revue de Linguistique romane 38, 400-417.

Robbe-Grillet A.(1981), Djinn, Paris, Éditions de Minuit.

Salaberry R. (1998), "The development of aspectual distinctions in L2 French classroom learning', Canadian Modern Language Review 54/4, 508-542.

Schogt H.G. (1964), 'L'aspect verbal en français et l'élimination du passé simple', Word 20/1, 1-17.

Van Vliet H.R. (1983), 'The disappearance of the French passé simple: a morphological and sociolinguistic study’, Word 34/2, 89-113. 